\title{
Increased pasal insulin secretion in Pdzd2-deficient mice
}

\author{
S.W. Tsang ${ }^{a}$, D. Shao ${ }^{b}$, K.S.E. Cheah ${ }^{a}$, K. Okuse ${ }^{b}$, P.S. Leung ${ }^{c}$, K.-M. Yao ${ }^{a, *}$ \\ a Department of Biochemistry, LKS Faculty of Medicine, The University of Hong Kong, Pokfulam, Hong Kong SAR, China \\ ${ }^{\mathrm{b}}$ Division of Cell \& Molecular Biology, Faculty of Natural Sciences, Imperial College London, London, UK \\ c School of Biomedical Sciences, Faculty of Medicine, The Chinese University of Hong Kong, Shatin, N.T., Hong Kong SAR, China
}

\section{A R T I C L E I N F O}

\section{Article history:}

Received 16 July 2009

Received in revised form

12 November 2009

Accepted 12 November 2009

\section{Keywords:}

Pdzd2

Gene-trap mutagenesis

ANS-1E

Glucose-stimulated insulin secretion

$\hat{\mathrm{K}}_{\text {ATP }}$ channel

\begin{abstract}
A B S T R A C T
Expression of the multi-PDZ protein Pdzd2 (PDZ domain-containing protein 2) is enriched in pancreatic islet $\beta$ cells, but not in exocrine or $\alpha$ cells, suggesting a role for Pdzd2 in the regulation of pancreatic $\beta$-cell function. To explore the in vivo function of Pdzd2, Pdzd2-deficient mice were generated. Homozygous Pdzd2 mutant mice were viable and their gross morphology appeared normal. Interestingly, Pdzd2-deficient mice showed enhanced glucose tolerance in intraperitoneal glucose tolerance tests and their plasma insulin levels indicated increased basal insulin secretion after fasting. Moreover, insulin release from mutant pancreatic islets was found to be twofold higher than from normal islets. To verify the functional defect in vitro, Pdzd2 was depleted in INS-1E cells using two siRNA duplexes. Pdzd2depleted INS-1E cells also displayed increased insulin secretion at low concentrations of glucose. Our results provide the first evidence that $\mathrm{Pdzd} 2$ is required for normal regulation of basal insulin secretion.
\end{abstract}

(c) 2009 Published by Elsevier Ireland Ltd.

\section{Introduction}

Insulin produced by pancreatic $\beta$ cells is the key hormone responsible for maintaining glucose homeostasis. The regulation of insulin exocytosis in $\beta$ cells is complicated and has a number of mechanisms involved (MacDonald et al., 2005; Rorsman et al., 2000; Straub and Sharp, 2002). Upon the challenge of a stimulus, e.g. glucose, exocytosis of insulin is triggered. Such glucose-stimulated insulin secretion (GSIS) is commonly distinguished as a biphasic process which depends on the rise of $\left[\mathrm{Ca}^{2+}\right]_{i}$. As the extracellular glucose concentration increases above $6 \mathrm{mM}$, glucose enters rodent $\beta$ cells through Glut2 transporters (Thorens et al., 1988). Subsequent degradation of glucose causes an elevation in the ATP/ADP ratio, and hence directs the closure of the $\mathrm{K}_{\mathrm{ATP}}$ channel. Followed by membrane depolarization, the influx of $\mathrm{Ca}^{2+}$ activates the exocytosis of insulin granules (Ashcroft and Rorsman, 1989; Ammala et al., 1993). As defective or deficient insulin secretory responses that result from impaired pancreatic $\beta$-cell functions lead to diabetes mellitus (Ashcroft, 2005; Koster et al., 2005), an understanding of the regulatory mechanisms of GSIS is definitely relevant to the pathogenesis and treatment of diabetes.

\footnotetext{
* Corresponding author at: Department of Biochemistry, LKS Faculty of Medicine, The University of Hông Kong, 3/F Laboratory Block, LKS Faculty of Medicine Building, 21 Sassoon Road, Pokfulam, Hong Kong SAR, China. Tel.: +852 2819 9275; fax: +852 28551254 .

E-mail address: kmyao@hkusua.hku.hk (K.-M. Yao).
}

Pdzd2 [previously named KIAA0300 (Nagase et al., 1997), PIN1 (Thomas et al., 1999), PAPIN (Deguchi et al., 2000), activated in prostate cancer (AIPC, Chaib et al., 2001)] is a multi-PDZ protein expressed in multiple tissues including heart, brain, lung and pancreas (Yeung et al., 2003). Immunohistochemical analyses revealed selective expression of Pdzd2 in pancreatic islet $\beta$ cells, but not in exocrine or glucagon-secreting $\alpha$ cells (Ma et al., 2006). Pdzd2 cDNAs were originally cloned based on the protein's binding and expression properties but its function remains obscure as no functional analysis in animal or cell model has been reported. Pdzd2 contains six different PDZ protein-protein interaction domains, which are thought to function by acting as molecular scaffolds to facilitate the assembly of macromolecular complexes (Nourry et al., 2003; Kim and Sheng, 2004; Harris and Lim, 2001). Pdzd2 shows the strongest sequence match to pro-interleukin-16 at the carboxy-terminus (Yeung et al., 2003; Baier et al., 1997) but is most structurally similar to the longer neuronal form of prointerleukin16 (npro-IL-16, Kurschner and Yuzaki, 1999), which is a 5 -PDZ domain protein. Npro-IL-16 has been reported to interact with the NMDA receptor subunit $2 \mathrm{~A}$ and Kv4 channels in interaction assays (Kurschner and Yuzaki, 1999). The interactions of npro-IL16 with Kv4.2 in the hippocampus and cerebellum were recently found to modulate Kv4.2 channel function (Fenster et al., 2007). The structural similarity between npro-IL-16 and Pdzd2 implies a potential intracellular role for $\mathrm{Pdzd} 2$, acting as a scaffolding protein for targeting receptors or jon channels. The purpose of this study was to establish mouse lines in which Pdzd2 is disrupted by genetrap mutagenesis in order to address the functional role of this large PDZ protein in the endocrine pancreas or $\beta$-cell physiology in vivo. 
In addition, silencing of Pdzd 2 expression in the $\beta$-cell-derived INS$1 \mathrm{E}$ line was performed using siRNAs to confirm functional relevance in vitro. Our results show that without affecting the expression of the $\mathrm{K}_{\text {ATP }}$ channel subunits, basal insulin secretion is distorted in Pdzd2-deficient mice as well as in the Pdzd2-depleted INS-1E cells and suggest that $\mathrm{Pdzd} 2$ is required in the regulation of insulin exocytosis through modulation of $\mathrm{K}_{\mathrm{ATP}}$ channel activity.

\section{Materials and methods}

\subsection{Generation of the gene-trap $\left(P d z d 2^{G t / G t}\right)$ mutant mice}

Two mouse ES lines, S3-7A and S7-9G, with the Pdzd2 locus mutated by genetrap mutagenesis, were purchased from Fred Hutchinson Cancer Research Center. These ES cells were used to generate chimeras by blastocyte (C57/B16) injection and germline transmission of the mutant allele was achieved by crossing the chimeras to $129 / \mathrm{SvEv}$ mice. Homozygous mutants (designated $\mathrm{Pdzd} 2^{\mathrm{Gt} / \mathrm{Gt}}-\mathrm{A}$ and $\mathrm{Pdzd} 2^{\mathrm{Gt} / \mathrm{Gt}}-\mathrm{B}$ ) were generated by intercrossing heterozygotes. To suppress transcription downstream of the sites of insertion of the retroviral gene-trap vector ROSAFARY (Chen et al., 2004), the PGK promoter flanked with FRT sites within ROSAFARY was removed by crossing with a mouse line that constitutively expressed the Flp recombinase. Mutant and wild-type mice were bred and housed in the Minimal Disease Area (MDA) unit at the University of Hong Kong. Sex- and age-matched mutants and their non-transgenic littermates were used in the present study. All animal procedures have been approved by the Committee on the Use of Live Animals in Teaching \& Research at the University of Hong Kong.

\subsection{Evaluation of glucose tolerance and plasma insulin}

Mice were starved for $14 \mathrm{~h}$ prior to the glucose challenge. Two grams of glucose per $\mathrm{kg}$ of body weight, in a $20 \%(\mathrm{w} / \mathrm{v})$ glucose solution, was injected into the peritoneal cavity of individual animals. Blood glucose levels of the mice were monitored $30 \mathrm{~min}$ before and 10,20, 30, 60 and $120 \mathrm{~min}$ after glucose injection using the Glucometer Elite (Bayor Inc.). As insulin levels Atere measured, blood samples were drawn from the animals 30 min prior to and 2.5, 5, 10, 15, 30 and 60 min after glucose injection using the Microvette CB300 lithium heparin-coated blood collection device (Sarstedt, Germany). Whole blood samples were subsequently centrifuged at $4600 \mathrm{rpm}$ for $5 \mathrm{~min}$ at room temperature. Plasma was collected and stored at $-20^{\circ} \mathrm{C}$ ûntil use. Plasma insulin le $\widehat{\text { els }}$ were measured utilizing an insulin ELISA kit $\widehat{\text { INCO }}$ Research, USA). For insulin tolerance tests, mice starved for $7 \mathrm{~h}$ were injected with insulin at $0.75 \mathrm{IU} / \mathrm{kg}$ and the blood glucose levels measured at the indicated time intervals.

\subsection{Islet isolation and culture}

Mice were sacrificed by cervical dislocation. The pancreatic tissue was harvested, rinsed and trimmed of fat, as previously described (Lau et al., 2004). Briefly, two pancreases were placed in $8 \mathrm{~mL}$ HBSS containing $25 \mathrm{mg}$ collagenase A (Roche) at $37^{\circ} \mathrm{C}$ for $25 \mathrm{~min}$ with vigorous shaking. The islets were handpicked under a light microscope and were cultured in RPMI 1640 medium. Typically, islets became free of other contaminating cell types after 3 days of incubation and were ready for different assays.

\subsection{INS-1E cell culture and RNAi}

Two pairs of the siRNA duplexes, designated as duplex2 (sense: $5^{\prime}$-UUC UCC GAA CGU GUC ACG UdTdT-3' and antisense: 5'-ACG UGA CAC GUU CGG AGA AdTdT-3' from Qiagen) and duplex3 (sense: 5'-GCU GCA GGA GUA CAU CCA AdTdT-3' and antisense: 5'-UUG GAU GUA CUC CUG CAG CdTdT-3' from Health \& Co.), were specifically designed for targeting the third and first PDZ domain, respectively, of rat Pdzd2, whereas one non-silencing siRNA duplex, duplex 1 (sense: 5'-UUC UCC GAA CGU GUC ACG UdTdT-3' and antisense: 5'-ACG UGA CAC GUU CGG AGA AdTdT-3' from Qiagen), was used as a negative control. siRNA oligos $(10 \mathrm{nM})$ were delivered into INS-1E cells using HiPerfect transfection reagent (Qiagen) according to the manufacturer's instructions. Samples were harvested $48 \mathrm{~h}$ after transfection.

\subsection{RT-PCR analysis}

Total RNA from islets or INS-1E cells was isolated in TRIzol (GIBCO) according to the manufacturer's instructions. Two micrograms of total RNA were reverse transcribed using random hexamers and SuperScript II Reverse Transcriptase (Invitrogen). Various mouse and rat cDNAs were amplified by PCR using SuperMix II (Invitrogen) and the conditions were as follows: an initial denaturation at $94^{\circ} \mathrm{C}$ for $1 \mathrm{~min}$; repeated cycles of denaturation at $94^{\circ} \mathrm{C}$ for $15 \mathrm{~s}$, annealing at $55-58^{\circ} \mathrm{C}$ for $30-45 \mathrm{~s}$ and extension at $68^{\circ} \mathrm{C}$ for $2 \mathrm{~min}$; and a final $\mathrm{PCR}$ cycle with an additional extension step at $68^{\circ} \mathrm{C}$ for $8 \mathrm{~min}$. All amplifications were performed for $20-40$ cycles and the number of cycles was optimized to fall within the linear rdnge for semi-quantitative analysis. For TaqMan-based real-time PCR analysis of Glut2 (Mm00446224_m1) and Insulin (Mm01950294_s1) transcripts, reactions were performed using the ABI 7500 Real-Time PCR system with the following therma cycle program: $5 \mathrm{~min}$ at $95^{\circ} \mathrm{C}$ followed by 40 cycles of $15 \mathrm{~s}$ at $95^{\circ} \mathrm{C}$ and $35 \mathrm{~s}$ at $60^{\circ} \mathrm{C}$. Reactions were performed in triplicate with gene expression normalized to glyceraldehyde-3-phosphate dehydrogenase and data analyzed using comparative $\mathrm{Ct}$ method. Details about primer design will be furnished upon request.

\subsection{Immunohistochemical, histomorphometric and western blot analyses}

Pancreatic tissues were fixed in $4 \%$ paraformaldehyde in PBS and cleared with xylene. Paraffin-embedded tissue blocks were sectioned into $6 \mu \mathrm{m}$ slices using the microtome machine. Slides were de-paraffinized, briefly rinsed with PBS and exposed to primary antibodies, anti-Pdzd2 [1:1000 for C-terminus antibody (Yeung et al., 2003); 1:2500 for N-terminus antibody], anti-insulin (pre-diluted, LINCO Research) and anti-glucagon ( $1: 2000$, DakoCytomation) for about $16 \mathrm{~h}$ at $4{ }^{\circ} \mathrm{C}$. After several washes with Tris-buffered saline containing $0.1 \%$ Tween-20 (TBS-T), the sections were incubated with HRP-, Cy3- or Cy5-conjugated anti-rabbit or anti-goat secondary antibodies at room temperature for $1 \mathrm{~h}$. For DAB staining, sections were covered with liquid $\mathrm{DAB}$ and substrate-chromben solution from the EnVision+ System-HRP (DAB) kit (DakoCytomation). The anti-Pdzd2 N-terminus antibody was raised against the peptide sequence CDTADDPSSELENGT (amino acids 240-254) identified within mouse Pdzd2 and was partially purified by the manufacturer (United States Biological). Islet area and islet mass were determined according to Skau et al. (2001).

Protein from INS-1E cells or islets was extracted in RIPA buffer [ $150 \mathrm{mM} \mathrm{NaCl}$, $1 \% \mathrm{NP}-40,0.5 \%$ deoxycholate, $1 \mathrm{mM}$ EDTA, $50 \mathrm{mM}$ Tris- $\mathrm{HCl}$ ( $\mathrm{pH} 7.4$ ), proteinase inhibitor cocktail (Roche Applied Science)] on ice. Samples were then solubilized in $5 \times$ SDS sample buffer [62.5 mM Tris- $\mathrm{HCl}$ (pH 6.8), $2 \%$ SDS, $5 \% \beta$-mercaptoethanol, $0.025 \%$ bromophenol blue, $20 \%$ glycerol]. Twenty micrograms of protein were resolved on SDS polyacrylamide gels (7.5-15\%), subjected to electrophoresis and transferred to Hybond-C membranes (Arfersham). Bound proteins were blocked with $5 \%$ non-fat dry milk in TBS-T at room temperature for $1 \mathrm{~h}$, and were probed with specific primary antibodies against Pdzd2 $(1: 1000,13)$, SUR̂ $(1: 250$, H-80 from Santa Cruz), Kir6.2 (1:250, N-18 from Santa Cruz) and $\alpha$-Tubulin (1:1000, NeoMarkers) overnight at $4{ }^{\circ} \mathrm{C}$. Membranes were then washed with TBS-T and incubated with appropriate HRP-conjugated secondary antibodies at room temperature for half an hour. Immunoreactive bands were visualized using an enfinced chemiluminescence detection system (Pierce).

\subsection{Assays for insulin secretion}

In a 24-well plate, INS-1E cells were seeded at a density of $4 \times 10^{4}$ or groups of 15 isolated mouse islets were placed (Lau et al., 2004). After pre-incubation in complete RPMI 1640 medium containing $2.8 \mathrm{mM}$ glucose for $4 \mathrm{~h}$, cells or islets were briefly washed with PBS. At time 0 , samples were incubated in $0.5 \mathrm{~mL}$ Krebs-Ringer bicarbonate (KRB) buffer supplemented with $2.8 \mathrm{mM}$ glucose for $30 \mathrm{~min}$ at $37^{\circ} \mathrm{C}$. The ambient buffer was then collected for the measurement of insulin secretion. At time $30 \mathrm{~min}$, samples were switched to be incubated in $0.5 \mathrm{~mL}$ KRB buffer containing $16.7 \mathrm{mM}$ glucose for another $30 \mathrm{~min}$ at $37^{\circ} \mathrm{C}$. Again, the ambient buffer was collected for determination of insulin secretion after glucose stimulation. Besides glucose, INS-1E cells and mouse islets were subjected to various stimuli, including $100 \mu \mathrm{M}$ tolbutamide (Sigma), $300 \mu \mathrm{M}$ diazoxide (Sigma) and $30 \mathrm{mM} \mathrm{KCl}$. Insulin concentrations in the collected samples were measured using an insulin ELISA kit (LINCO Research) and were normalized to the corresponding insulin content.

\subsection{Statistical analyses}

The data are presented as means \pm S.E.M. Significance was evaluated by unpaired Student's $t$-test or one-way analysis of variance (ANOVA) with appropriate post hoc tests. Statistical significance by either unpaired Student's $t$-test or ANOA is explicitly identified by text or in the figure legends.

\section{Results}

\subsection{Pdzd2 expression is disrupted in two mouse gene-trap lines}

Both Pdzd2 heterozygous and homozygous mutants derived from the two gene-trap lines were viable and appeared grossly morphologically normal. To avoid extensive hormonal effects in physiological assays, male animals were used in experiments and only wild-type and homozygous mutant mice were studied to clearly detect any loss-of-function phenotype. The viral insertion sites of both $P d z d 2^{G t / G t}-A$ and $P d z d 2^{G t / G t}-B$ lines are downstream of the first coding exon (Exon 1) of $P d z d 2$ and they are about 35,000 bp apart (Fig. 1A). RT-PCR analysis of $P d z d 2$ transcripts revealed that splicing from Exon 1 to Exon 2 was disrupted in the gene-trap mutants of both lines (Fig. 1B, Primers 1 and 2). As a result, transcription across Exon 2 and Exon 3 was also halted (Fig. 1B, Primers 3 and 4). Next, we examined the protein levels of Pdzd2 

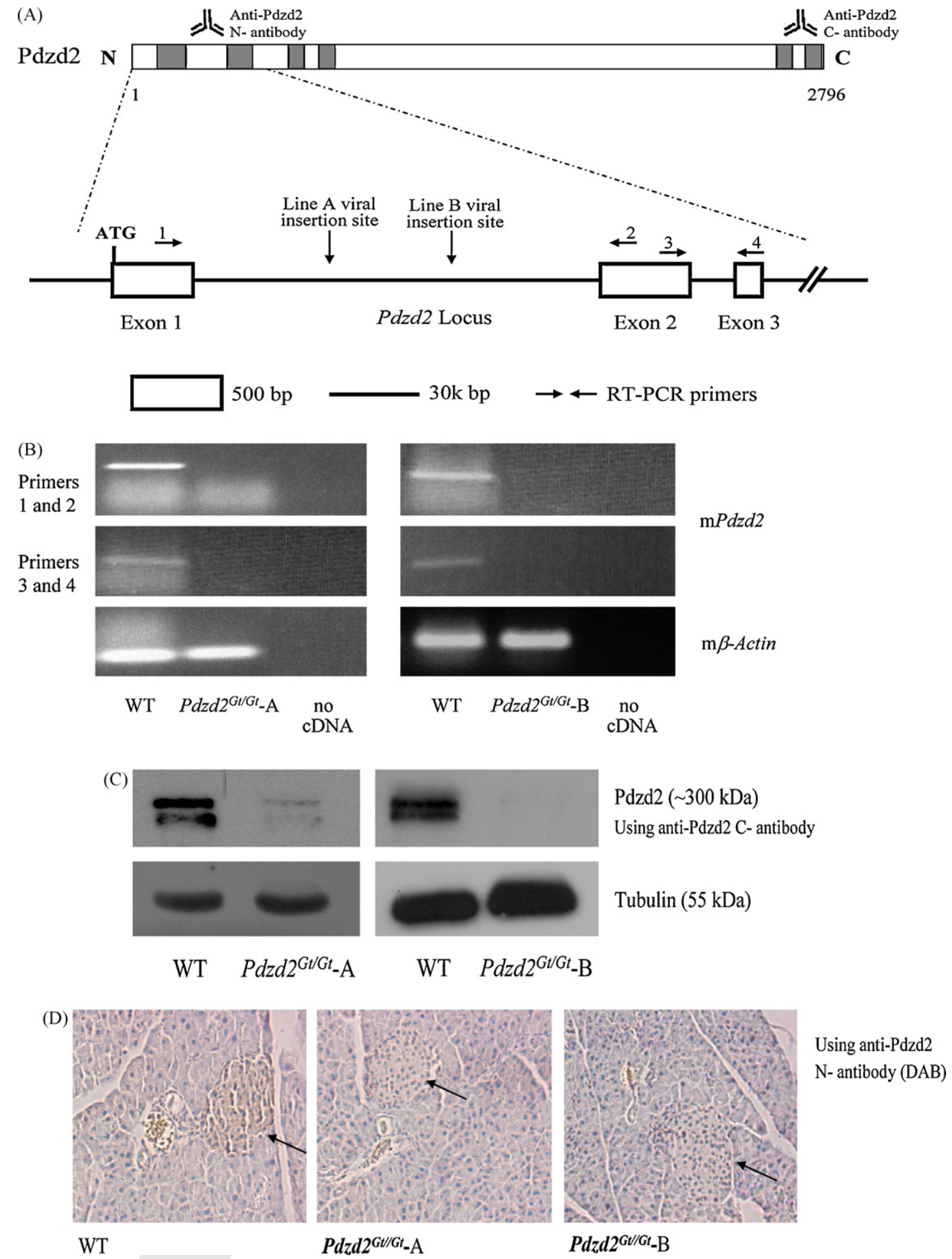

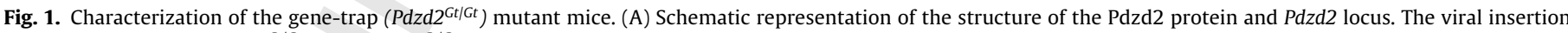

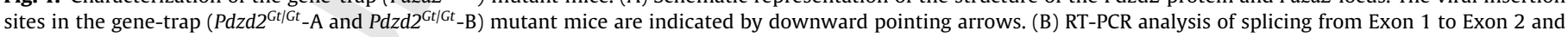

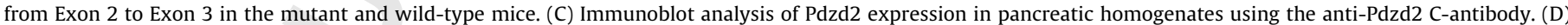

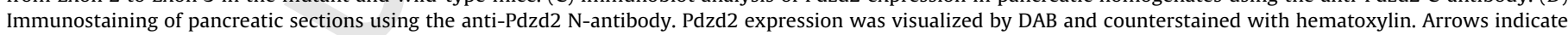
the position of islets. Magnification 200x.

in pancreatic tissues by immunoblot analysis utilizing an antiPdzd2 C-terminus antibody. The expression of Pdzd2 ( $300 \mathrm{kDa})$ in the gene-trap mutant pancreases was suppressed when compared with those from the wild-type mice (Fig. 1C). The disruption in expression of this large Pdzd2 protein was further confirmed by immunostaining using an anti-Pdzd2 N-terminus antibody (Fig. 1D). This analysis confirmed the islet-enriched expression of Pdzd2 that was lost in the gene-trap mutant pancreases.

\subsection{Pdzd2 deficiency has no detectable effect on islet morphology or expression of important $\beta$-cell markers}

Immunohistochemical studies were performed using antibodies against the major pancreatic endocrine hormones, insulin and glucagon, to identify $\alpha$ and $\beta$ cells. Pancreatic sections from wild-type and $P d z d 2^{G t / G t}-A$ mutants were found to contain the two islet cell types and no notable alteration was observed in 

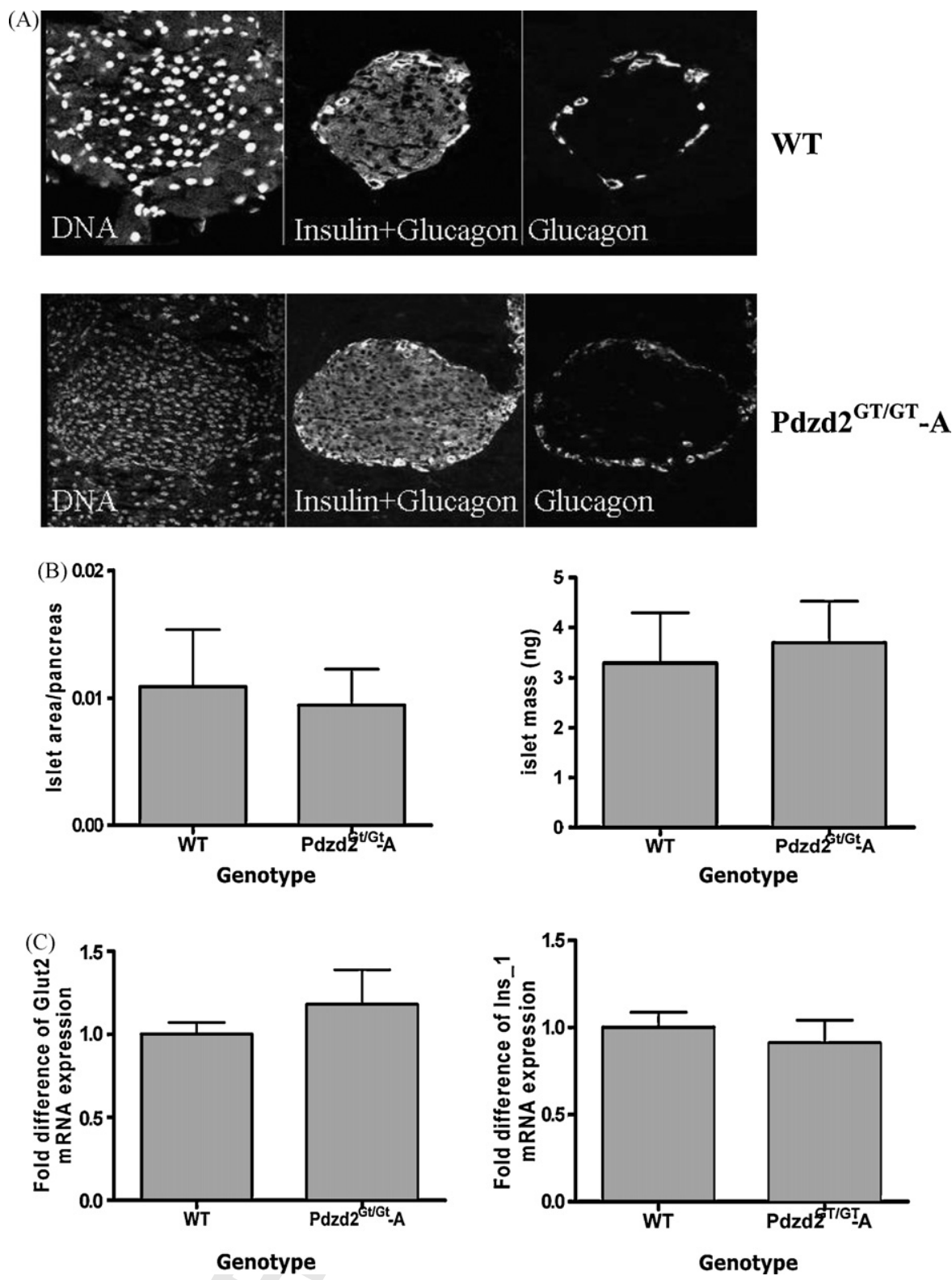

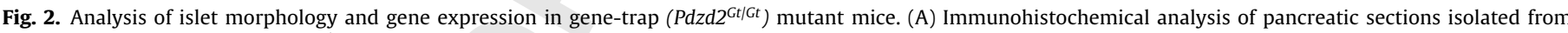

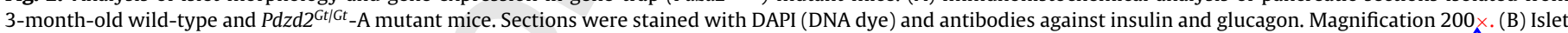

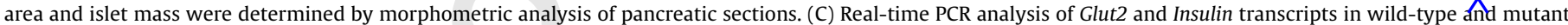
pancreases. Data are means \pm S.E. of values from at least three mice of each genotype.

their localization (Fig. 2A). In addition, histomorphometric analysis of insulin-expressing cells did not reveal any significant difference in islet mass or islet area/pancreas in Pdzd2 $2^{G t / G t}-\mathrm{A}$ mutants (Fig. 2B) and the mRNA expression of two important $\beta$-cell markers, Insulin and Glut2, was not significantly affected by Pdzd2 deficiency (Fig. 2C). Similar results were also obtained from $P d z d 2^{G t / G t}-B$ mutants (data not shown). Hence, Pdzd2 deficiency does not appear to have any negative effect on the formation of the different islet cell types, general islet morphology and $\beta$-cell mass.

\subsection{Pdzd2 $2^{G t / G t}$ mutant mice have slightly lower body weight and show enhanced glucose tolerance}

At the ages of 2 and 3 months, the body weight of $P d z d 2^{G t / G t}$ mutants, from both lines $\mathrm{A}$ and $\mathrm{B}$, was found to be lower when compared with the wild-type mice (Fig. 3A). Moreover, Pdzd2 ${ }^{G t / G t}$ mutants exhibited a relative lower level of blood glucose in the random-fed state (Fig. 3B), along with a slightly higher plasma level of insulin (Fig. 3C). However, those differences were not regarded as statistically significant. To investigate possible change in glucose homeostasis in the $P d z d 2^{G t / G t}$ mice, an intraperitoneal injection of glucose at $2 \mathrm{~g} / \mathrm{kg}$ of body weight was given to animals (at 1,2 and 3 months of age) that had been fasted overnight. Interestingly, $\mathrm{Pdzd} 2^{\mathrm{Gt} / \mathrm{Gt}}$ mutants of both lines A and B at 2 and 3 months of age showed a faster clearance of blood glucose in contrast to wild-type mice; the differences were most significant at 20 and 30 min after glucose injection (Fig. 4B and C). However, such a phenomenon was not observed when the animals were only 1 -month old, suggesting a delayed onset of phenotype (Fig. 4A). The faster glucose clearance could not be attributed to increased insulin sensitivity of peripheral tissues as insulin tolerance tests revealed comparable responses in wild-type and $P d z d 2^{G t / G t}$-A mutants (Supplementary Fig. 1). 

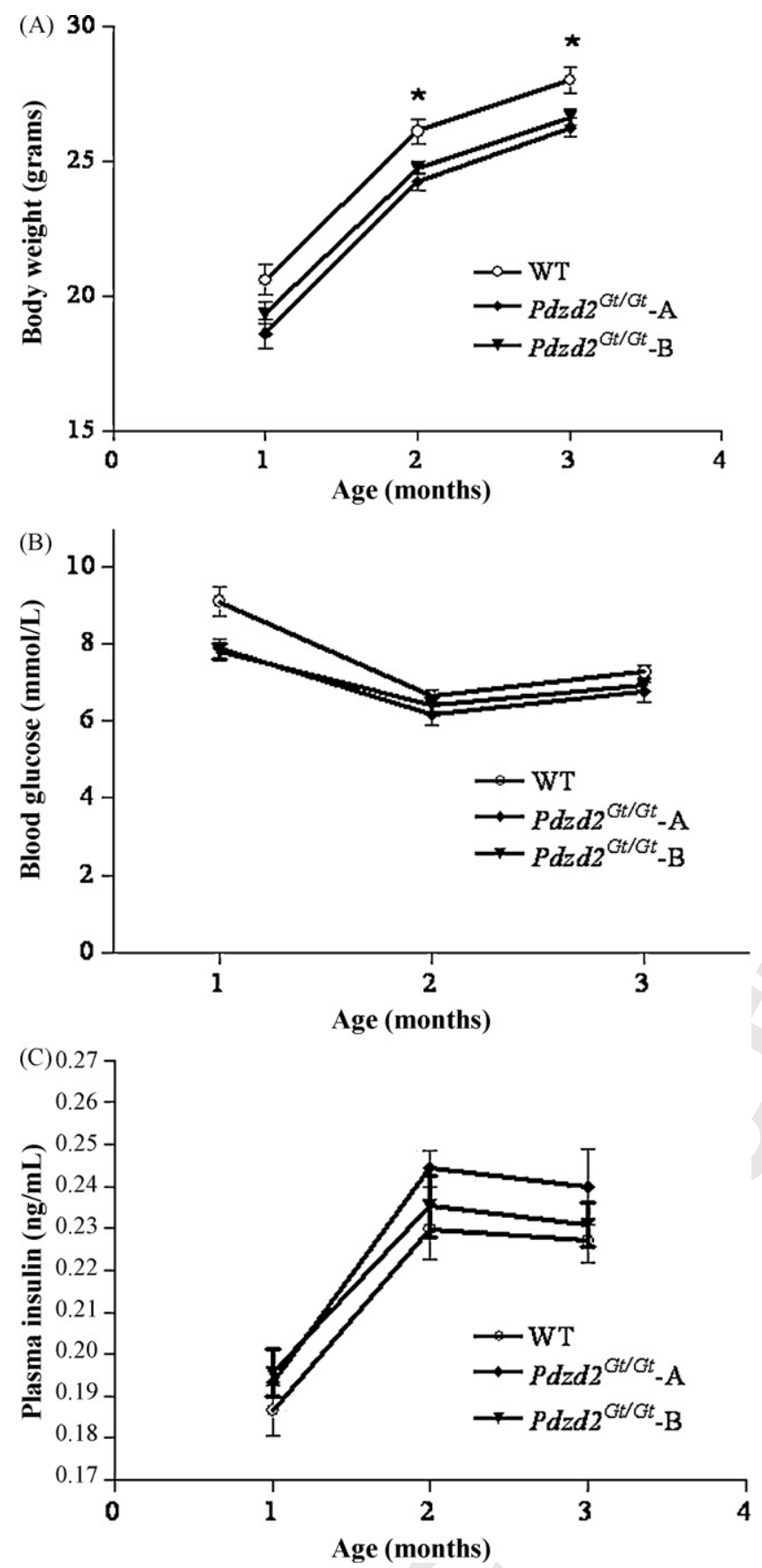

Fig. 3. $P d z d 2^{G t / G t}$ mutant mice have lower body weight. The body weight (A), fedstate blood glucose level (B) and fed-state plasma insulin level (C) were monitored when mice were 1, 2 and 3 months old. Data are means \pm S.E. of values from $10(A)$, 7 (B) or 4 (C) mice of each genotype. ${ }^{*} p<0.05$.
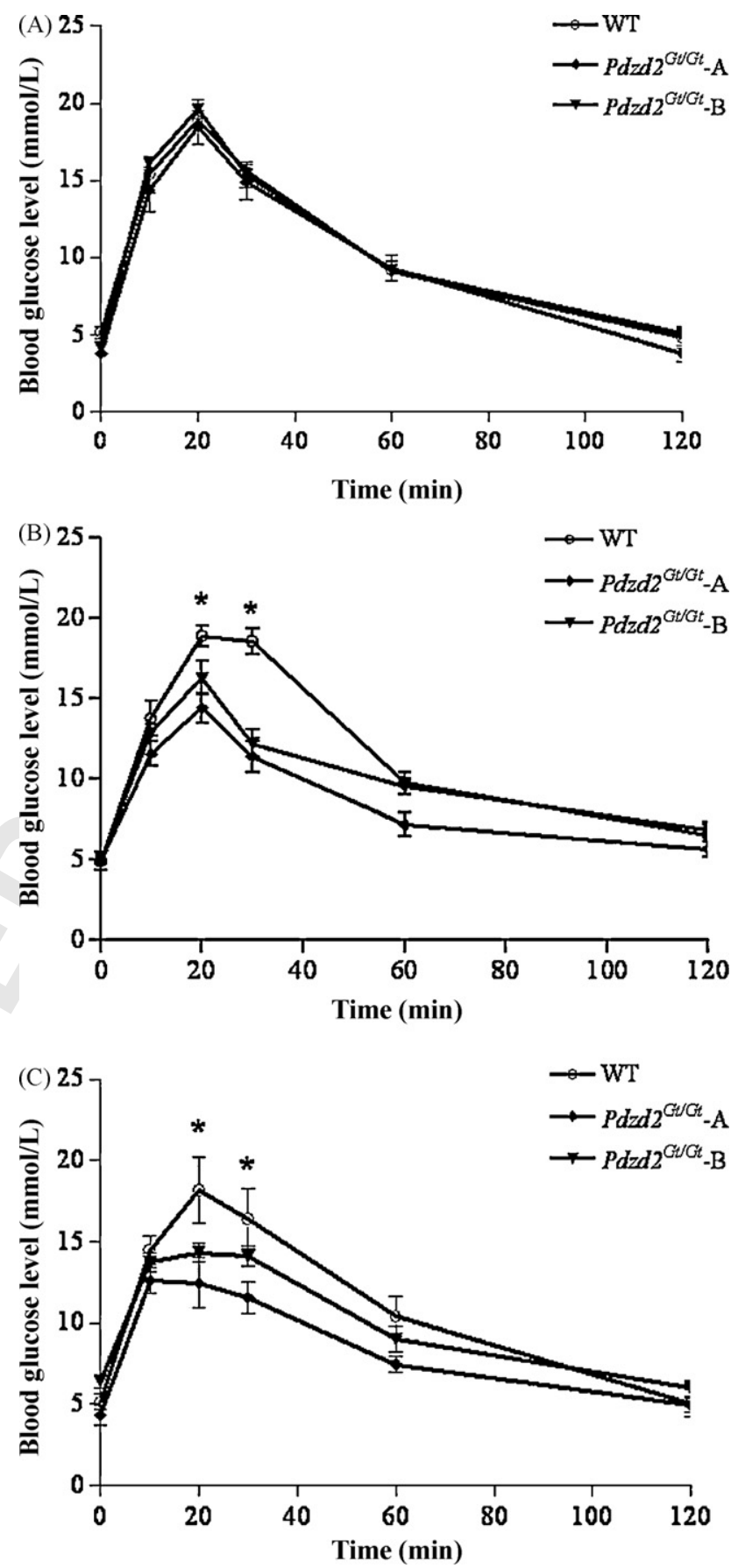

Fig. 4. Effect of Pdzd2 depletion on glucose clearance. Intraperitoneal glucose tolerance tests were performed in wild-type and $\mathrm{Pdzd} 2^{\mathrm{Gt} / \mathrm{Gt}}$ mice at 1 month (A), 2 months (B) and 3 months $(C)$ of age after fasting for 14 h. Data are means \pm S.E. of values from 8 (A) or 6 (B and C) mice of each genotype. ${ }^{*} p<0.05$.

continued higher level for the mutant mice at 10 and $15 \mathrm{~min}$. The wild-type mice then showed a second peak at 30 min which was absent in the mutant mice. Nevertheless, we cannot rule out the possibility of an early or delayed second peak without measurement of insulin secretion at more time points (between $T=15$ and $T=60$ ). These findings indicated that $P d z d 2^{G t / G t}$ mutants exhibited a significantly stronger basal level of insulin release.

\subsection{Insulin secretion is abnormal in isolated mutant islets}

To further investigate the effect of Pdzd2 deficiency on insulin secretion, glucose- or $\mathrm{KCl}$-stimulated insulin release with islets iso- 

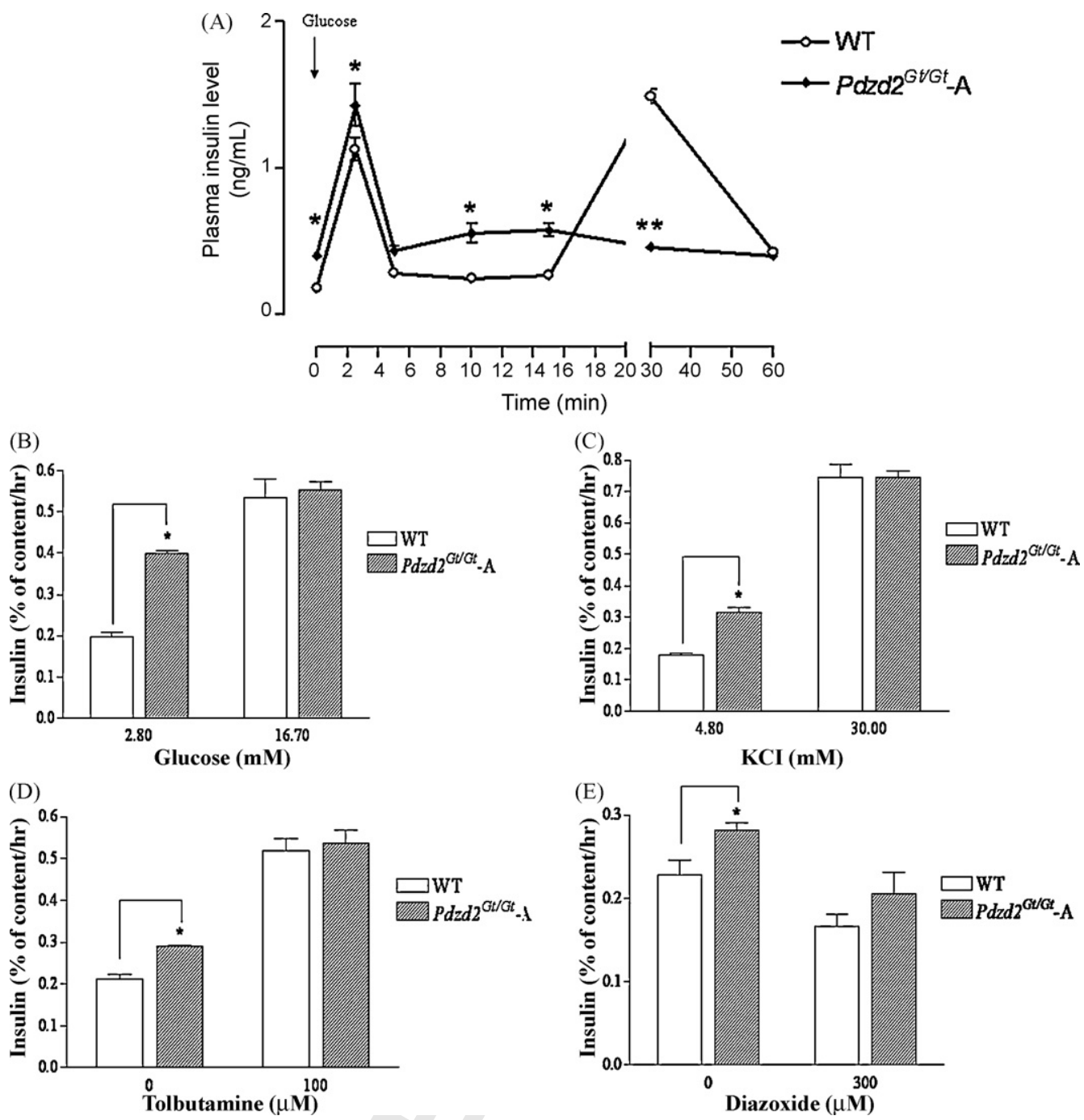

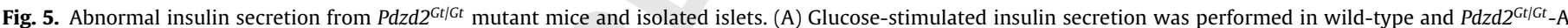

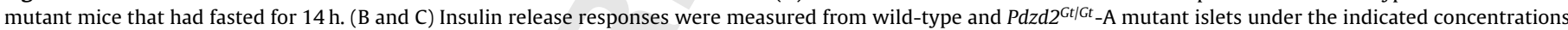

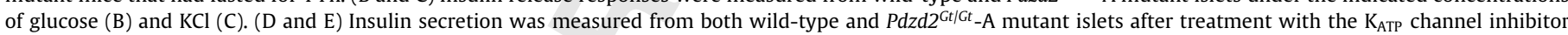

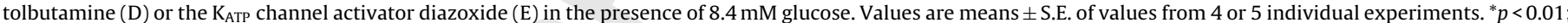
${ }^{* *} p<0.001$.

lated from $P d z d 2^{G t / G t}$-A mutant and wild-type mice was monitored. For such experiments, isolated islets were subdivided into groups of 15 with size-matching. At low concentration of glucose $(2.8 \mathrm{mM})$, insulin release from $P d z d 2^{G t / G t}$ islets was about twofold higher than from normal islets (Fig. 5B). At $16.7 \mathrm{mM}$ glucose, insulin secretion from both $P d z d 2^{G t / G t}$ and wild-type islets was essentially the same. $\mathrm{KCl}$ at $30 \mathrm{mM}$ is known to elicit insulin release by inducing membrane depolarization. The secretory response of the mutant islets to $4.8 \mathrm{mM} \mathrm{KCl}$ was higher than that of the normal islets, despite their similar level of response to $30 \mathrm{mM} \mathrm{KCl}$ (Fig. 5C). These results indicated that Pdzd2 is required for normal insulin secretion and disruption of Pdzd2 expression perturbed the control of basal insulin release consistent with the plasma insulin analysis.

The $K_{\text {ATP }}$ channel is known to be the key regulator of insulin secretion by maintaining hyperpolarization of $\beta$ cells and it is the closure of the $\mathrm{K}_{\text {ATP }}$ channel by increased ATP levels after glucose intake that triggers depolarization and eventually insulin secretion. RT-PCR and western analyses showed that the expression levels of the $K_{\text {ATP }}$ channel subunits were not suppressed by Pdzd2 deficiency (data not shown). To investigate the functional involvement of the $K_{A T P}$ channel in mediating the Pdzd2-dependent defect, the effects of tolbutamine $(100 \mu \mathrm{M})$ and diazoxide $(300 \mu \mathrm{M})$ which are inhibitor and activator, respectively, of $\mathrm{K}_{\text {ATP }}$ channel activity were tested. We argue that if the Pdzd2 effect were mediated via regulation of $\mathrm{K}_{\mathrm{ATP}}$ channel activity, the Pdzd2-dependent defect on insulin secretion would be lost if $\mathrm{K}_{\text {ATP }}$ channel activity was suppressed by tolbutamine treatment. Indeed, when tolbutamine was applied, insulin release from both wild-type and mutant islets was increased to comparable levels (Fig. 5D). In contrast, when the $K_{\text {ATP }}$ channel activator diazoxide was present, insulin release from both wild-type and mutant islets was decreased but the insulin secretion from mutant islets was still maintained higher (Fig. 5E), suggesting that the Pdzd2-dependent effect is mediated through regulation of $\mathrm{K}_{\text {ATP }}$ channel activity.

\subsection{Silencing of Pdzd2 expression in INS-1E cells produces similar} phenotypic effect as the mutant islets

To study the function of Pdzd2 in vitro, we designed two siRNA duplexes (duplexes 2 and 3) for silencing Pdzd2 expression by transient transfection into the $\beta$-cell-derived INS-1E cell line. A non-silencing duplex (duplex 1 ) Was also analyzed in parallel. RTPCR and immunoblot analyses revealed that Pdzd 2 at both RNA and protein levels was depleted by both silencing duplexes $48 \mathrm{~h}$ after 
(A)

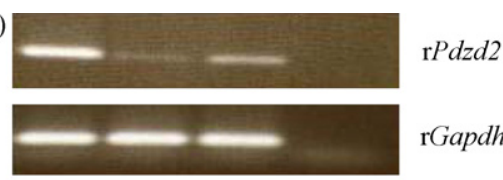

duplex1 duplex2 duplex3 no

cDNA
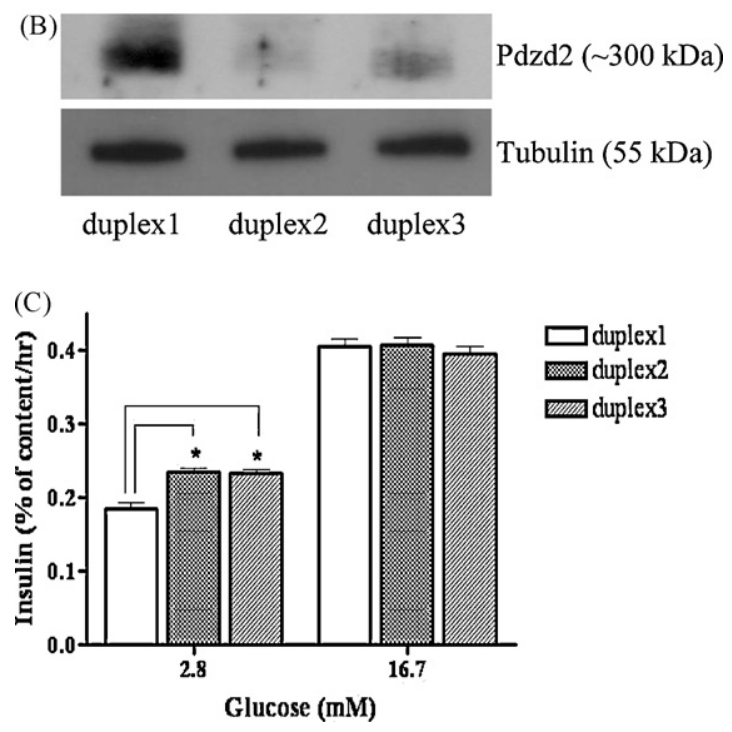

Fig. 6. Knockdown of Pdzd2 expression in INS-1E cells. (A and B) Silencing effects of different siRNA duplexes on Pdzd2 expression were examined by means of RT-PCR (A) and western analyses (B). Duplex1 is the non-silencing control. (C) Glucosestimulated insulin secretion. In the siRNA-transfected INS-1E cells, insulin release in response to indicated concentrations of glucose was measured by means of ELISA. Data are means \pm S.E. of values from 4 separate experiments. ${ }^{*} p<0.05$.

transfection (Fig. 6A and B). To further explore the effect of Pdzd2 silencing on insulin secretion, transfected cells were subjected to GSIS assays. Insulin secretion was measured using ELISA after incubation for $1 \mathrm{~h}$ in both basal $(2.8 \mathrm{mM})$ and stimulatory $(16.7 \mathrm{mM})$ doses of glucose. Similar to the ex vivo results, INS-1E cells under knockdown conditions secrete $\hat{A}$ nearly $20 \%$ more insulin even at the non-stimulatory concentration of glucose $(2.8 \mathrm{mM})$. In contrast, their responses to $16.7 \mathrm{mM}$ glucose were comparable to the control cells (Fig. 6C). When transfected INS-1E cells were similarly treated with tolbutamine and diazoxide to study the involvement of the $\mathrm{K}_{\text {ATP }}$ channel, the effect of Pdzd2 knockdown was abolished when $K_{\text {ATP }}$ channel activity was inhibited (Supplementary Fig. 2B) but not when the channel was activated (Supplementary Fig. 2C).

Taken together, these results suggested that insulin secretion was distorted by Pdzd2 deficiency. At low concentrations of glucose, basal insulin secretion of both Pdzd2-deficient islets and INS-1E cells was abnormally increased. The difference in response of wild-type and mutant islets to tolbutamine and diazoxide, and similarly for normal and Pdzd2-depleted INS-1E cells, suggested that the enhanced insulin exocytosis was primarily mediated by suppression of $\mathrm{K}_{\mathrm{ATP}}$ channel activity.

\section{Discussion}

In this study, we utilized gene-trap mutagenesis to uncover a functional role of Pdzd2 in insulin secretion and glucose homeostasis. The physiological defect was very evident in intraperitoneal glucose tolerance tests when $P d z d 2^{G t / G t}$ mutant mice exhibited an unusually faster clearance of glucose. $P d z d 2^{G t / G t}$ mutant mice developed with an elevated basal level of plasma insulin in the fasting state. The functional defect could be observed in two independent mouse lines with the mutagenic retrovirus inserted $35 \mathrm{~kb}$ apart within the first intron of the Pdzd2 locus, ruling out the possibility of a secondary effect. Since enhanced basal level of insulin release was reproduced in $P d z d 2^{G t / G t}$ mutant islets in ex vivo assays and insulin tolerance tests argued against a significant change in insulin sensitivity in peripheral tissues, the physiological response of insulin secretion in mutant mice was attributable to the pancreatic islets. Without any obvious change in composition of various pancreatic endocrine cell types, the increased secretion of insulin could not be explained by changes in cell mass of individual cell types.

In normal pancreatic $\beta$ cells, basal release of insulin is tightly suppressed when the ambient glucose concentration is low. The release of insulin granules is known to be controlled by several mechanisms, and the $K_{\text {ATP }}$ channel is one of the most important governing gates (Straub and Sharp, 2002). The $K_{\text {ATP }}$ channel exists as an octameric complex of 2 subunits, SUR1 and Kir6.2, and its activity is thereby regulated by these two proteins (Ashcroft and Gribble, 1999). Mice lacking Kir6.2 developed transient hypoglycemia as insulin secretion was not suppressed at low concentrations of glucose (Seino et al., 2000), and suppression of beta cell $\mathrm{K}_{\mathrm{ATP}}$ channel activity was found to induce hyperinsulinism (Koster et al., 2002). In the current study, $P d z d 2^{G t / G t}$ mutant mice showed an elevated level of plasma insulin during fasting and insulin secretion in $P d z d 2^{G t / G t}$ mutant islets was not sufficiently suppressed at $2.8 \mathrm{mM}$ glucose, consistent with a perturbation of $\mathrm{K}_{\text {ATP }}$ channel activity. By means of RNAi to deplete Pdzd2 expression in INS-1E cells, abnormal insulin responses were observed. The $\mathrm{K}_{\mathrm{ATP}}$ channel being a target of Pdzd2 action is also supported by the differential response of mutant islets and Pdzd2-depleted INS-1E cells to tolbutamine and diazoxide. It is interesting to note that comparable levels of insulin secretion at $16.7 \mathrm{mM}$ glucose were observed in islets isolated from both wild-type and mutant islets. These findings are supportive of an early insulin release in the mutant mice that led to faster glucose clearance while the total amount of secreted insulin remained unaffected. Based on these in vivo and in vitro studies, it is plausible that Pdzd2 takes part in the regulation of insulin secretion via modulation of $\mathrm{K}_{\mathrm{ATP}}$ channel activity. Without any apparent decrease in the expression of the $\mathrm{K}_{\mathrm{ATP}}$ channel subunits, we believe that it was the functional activity rather than the expression level of the $\mathrm{K}_{\mathrm{ATP}}$ channel that was affected in the mutant islets.

As a multi-PDZ domain protein, it is conceivable that Pdzd2 may modulate channel activity by serving as a molecular scaffold to mediate the formation of multi-molecular complexes (Nourry et al., 2003; Kim and Sheng, 2004; Harris and Lim, 2001). One of the highly comparable PDZ domain proteins, npro-IL-16, has been reported to interact physically with the NMDA receptor subunit 2A and Kv4 channels (Kurschner and Yuzaki, 1999). A recent study has shown that npro-IL-16 modulates A-type potassium channel function via regulating channel trafficking in the hippocampus and cerebellum (Fenster et al., 2007). Npro-IL-16 regulates the surface expression of Kv4.2 by altering its intracellular clustering, without any effect on Kv4.2 protein expression levels. Since Pdzd2 is required for suppressing insulin exocytosis, it may function to enhance the intracellular trafficking and hence surface expression of the $\mathrm{K}_{\mathrm{ATP}}$ channel in pancreatic $\beta$ cells. Further interaction assays need to be performed to test whether Pdzd2 interacts directly with the $\mathrm{K}_{\mathrm{ATP}}$ channel subunit SUR1 and Kir6.2. It remains to be tested whether Pdzd2 would also interact with signaling lipids (Zimmermann, 2006) to mediate its regulatory function. It is worth noting that $\operatorname{Pdzd} 2$ has recently been predicted to interact with a variety of ion channels in a large-scale analysis of PDZ interactions (Stiffler et al., 2007). Consistent with this prediction, Shao et al. (2009) recently detected a direct interaction of Pdzd2 with the voltage-gated sodium channel $\mathrm{Na}_{\mathrm{v}} 1.8$ and siRNA-depleted Pdzd2 expression in sensory neurons led to inhibition of endogenous $\mathrm{Na}_{\mathrm{v}} 1.8$ current. Interestingly, no significant change in pain behav- 
iors could be detected when the Pdzd2-deficient mice reported in this study were analyzed for pain sensation, probably due to the compensatory upregulation of another $\mathrm{Na}_{\mathrm{v}} 1.8$ regulatory factor p11 (Shao et al., 2009). Mouse models for conditional knockout of Pdzd2 would need to be established to further study the tissueor cell type-specific function of Pdzd2.

Hitherto, studies of Pdzd2 function have mostly been focused on the secreted form generated by caspase-dependent proteolytic processing of the full-length form (Yeung et al., 2003; Thomas et al., 2009). In a concentration-dependent fashion, recombinant sPDZD2 has been shown to stimulate the proliferation and differentiation of INS-1E cells in vitro (Ma et al., 2006; Suen et al., 2008; Leung et al., 2009). Although it is surprising to note a lack of perturbation of $\beta$ cell mass and gene expression in the Pdzd2 mutant mice, our study revealed a role for Pdzd2 in the regulation of basal insulin secretion. In summary, Pdzd2 depletion leads to increased basal release of insulin. The tight suppression of insulin release is lost suggesting that $\mathrm{Pdzd} 2$ is required for activation of the $\mathrm{K}_{\mathrm{ATP}}$ channel. The identification of putative interactors of $\mathrm{Pdzd} 2$ may provide further insight into the molecular basis of Pdzd2 function. Since dysfunction of the $\mathrm{K}_{\mathrm{ATP}}$ channel and insulin secretion has been implicated in the development of diabetes, it would also be interesting to know whether the defective insulin secretion in Pdzd2-deficient mice leads to diabetes or impaired glucose tolerance with aging or diet-induced obesity.

\section{Acknowledgements}

This work was supported by grants from the Research Grants Council, HKSAR, China and the University Grants Committee of the University of Hong Kong awarded to K.-M. Yao (HKU 7474/04M and Small Project Fund) and K. Cheah (AoE/M-04/04). We thank S. Fu, W. Chan (Transgenic Core Facility, The University of Hong Kong) and S. Tsang for ES cell culture, blastocyte injections and animal husbandry, and Y. Wang, J. Chan and W.-Y. Leung for their help in various analyses. We are grateful to M. K. Thomas, K. Chan and J. C. Y. Chan for their critical review of this manuscript.

\section{Appendix A. Supplementary data}

Supplementary data associated with this article can be found, in the online version, at doi:10.1016/j.mce.2009.11.007.

\section{References}

Ammala, C., Eliasson, L., Bokvist, K., Larsson, O., Ashcroft, F.M., Rorsman, P., 1993. Exocytosis elicited by action potentials and voltage-clamp calcium currents in individual mouse pancreatic B-cells. J. Physiol. 472, 665-688.

Ashcroft, F.M., 2005. ATP-sensitive potassium channelopathies: focus on insulin secretion. J. Clin. Invest. 115, 2047-2058.

Ashcroft, F.M., Gribble, F.M., 1999. ATP-sensitive K+ channels and insulin secretion: their role in health and disease. Diabetologia 42, 903-919.

Ashcroft, F.M., Rorsman, P., 1989. Electrophysiology of the pancreatic beta-cell. Prog. Biophys. Mol. Biol. 54, 87-143.

Baier, M., Bannert, N., Werner, A., Lang, K., Kurth, R., 1997. Molecular cloning, sequence, expression, and processing of the interleukin 16 precursor. Proc. Natl. Acad. Sci. U.S.A. 94, 5273-5277.

Chaib, H., Rub今in, M.A., Mucci, N.R., Li, L., Taylor, J.M.G., Day, M.L., Rhim, J.S., Macoska, J.A., 2001. Activated in prostate cancer: a PDZ domain-containing protein highly expressed in human primary prostate tumors. Cancer Res. 61, 2390-2394.
Chen, W.V., Delrow, J., Corrin, P.D., Frazier, J.P., Soriano, P., 2004. Identification and validation of PDGF transcriptional targets by microarray-coupled gene-trap mutagenesis. Nat. Genet. 36, 304-312.

Deguchi, M., Iizuka, T., Hata, Y., Nishimura, W., Hirao, K., Yao, I., Kawabe, H., Takai, Y., 2000. PAPIN. A novel multiple PSD-95/Dlg-A/ZO-1 protein interacting with neural plakophilin-related armadillo repeat protein/ $\delta$-catenin and p0071.J. Biol. Chem. 275, 29875-29880.

Ferßter, C.P., Fenster, S.D., Leahy, H.P., Kurschner, C., Blundon, J.A., 2007. Modulation of Kv4.2 K+ currents by neuronal interleukin-16, a PDZ domain-containing protein expressed in the hippocampus and cerebellum. Brain Res. 1162, 19-31.

Harris, B.Z., Lim, W.A., 2001. Mechanism and role of PDZ domains in signaling complex assembly. J. Cell Sci. 114, 3219-3231.

Kim, E., Sheng, M., 2004. PDZ domain proteins of synapses. Nat. Rev. Neurosci. 5 771-781.

Koster, J.C., Permutt, M.A., Nichols, C.G., 2005. Diabetes and insulin secretion: the ATP-sensitive K+ channel (K ATP) connection. Diabetes 54, 3065-3072.

Koster, J.C., Remedi, M.S., Flagg, T.P., Johnson, J.D., Markova, K.P., Marshall, B.A. Nichols, C.G., 2002. Hyperinsulinism induced by targeted suppression of beta cell $K_{\text {ATP }}$ channels. Proc. Natl. Acad. Sci. U.S.A. 99, 16992-16997.

Kurschner, C., Yuzaki, M., 1999. Neuronal Anterleukin-16 (NIL-16): a dual function PDZ domain protein. J. Neurosci. 19, 7770-7780.

Lau, T., Carlsson, P.O., Leung, P.S., 2004. Evidence for a local angiotensin-generating system and dose-dependent inhibition of glucose-stimulated insulin release by angiotensin II in isolated pancreatic islets. Diabetologia 47, 240-248.

Leung, K.K., Suen, P.M., Lau, T.K., Ko, W.H., Yao, K.M., Leung, P.S., 2009. PDZ-domain containing-2 (PDZD2) drives the maturity of human fetal pancreatic progenitorderived islet-like cell clusters with functional responsiveness against membrane depolarization. Stem Cells Dev. 18, 979-989.

Ma, R.Y., Tam, T.S., Suen, A.P., Yeung, P.M., Tsang, S.W., Chung, S.K., Thomas, M.K., Leung, P.S., Yao, K.M., 2006. Secreted PDZD2 exerts concentration-dependent effects on the proliferation of INS-1E cells. Int. J. Biochem. Cell Biol. 38 1015-1022.

MacDonald, P.E., Joseph, J.W., Rorsman, P., 2005. Glucose-sensing mechanisms in pancreatic beta-cells. Philos. Trans. R. Soc. Lond. B Biol. Sci. 360, 2211-2225.

Nagase, T., Ishikawa, K., Nakajima, D., Ohira, M., Seki, N., Miyajima, N., Tanaka, A. Kotani, H., Nomura, N., Ohara, O., 1997. Prediction of the coding sequences of unidentified human genes. VII. The complete sequences of 100 new cDNA clones from brain which can code for large protein in vitro. DNA Res. 4, 141-150.

Nourry, C., Grant, S.G., Borg, J.P., 2003. PDZ domain proteins: plug and play! Sci. STKE RE7.

RorShan, P., Eliasson, L., Renstrom, E., Gromada, J., Barg, S., Gopel, S., 2000. The cell physiology of biphasic insulin secretion. News Physiol. Sci. 15, 72-77.

Seino, S., Iwanaga, T., Nagashima, K., Miki, T., 2000. Diverse roles of K(ATP) channels learned from Kir6.2 genetically engineered mice. Diabetes 49, 311-318.

Shao, D., Baker, M.D., Abrahamsen, B., Rugiero, F., Malik-Hall, M., Poon, W.Y.L., Cheah, K.S.E., Yao, K.M., Wood, J.N., Okuse, K., 2009. A mult PDZ-domain protein Pdzd2 contributes to functional expression of sensory neuron-specific sodium channel $\mathrm{Na}_{\mathrm{v}}$ 1.8. Mol. Cell. Neurosci. 42, 219-225.

Skau, M., Pakkenberg, B., Buschard, K., Bock, T., 2001. Linear correlation between the total islet mass and the volume-weighted mean islet volume. Diabetes 50, 1763-1770.

Stiffler, M.A., Chen, J.R., Grantcharova, V.P., Lei, Y., Fuchs, D., Allen, J.E., Zaslavskaia, L.A., MacBeath, G., 2007. PDZ domain binding selectivity is optimized across the mouse proteome. Science 317, 364-369.

Straub, S.G., Sharp, G.W., 2002. Glucose-stimulated signaling pathways in biphasic insulin secretion. Diabetes Metab. Res. Rev. 18, 451-463.

Suen, P.M., Zou, C., Zhang, Y.A., Lau, T.K., Chan, J., Yao, K.M., Leung, P.S., 2008. PDZdomain containing-2 (PDZD2) is a novel factor that affects the growth and differentiation of human fetal pancreatic progenitor cells. Int. J. Biochem. Cel Biol. 40, 789-803.

Thomas, M.K., Tsang, S.W., Yeung, M.L., Leung, P.S., Yao, K.M., 2009. The roles of the PDZ-containing proteins Bridge-1 and PDZD2 in the regulation of insulin production and pancreatic beta-cell mass. Curr. Protein Pept. Sci. 10, 30-36.

Thomas, M.K., Yao, K.M., Tenser, M.S., Wong, G.G., Habener, J.F., 1999. Bridge-1, a novel PDZ-domain interactor of E2A-mediated regulation of insulin gene transcription. Mol. Cell Biol. 19, 8492-8504.

Thorens, B., Sarkar, H.K., Kaback, H.R., Lodish, H.F., 1988. Cloning and functiona expression in bacteria of a novel glucose transporter present in liver, intestine, kidney, and beta-pancreatic islet cells. Cell 55, 281-290.

Yeung, M.L., Tam, T.S., Tsang, A.C., Yao, K.M., 2003. Proteolytic cleavage of PDZD2 generates a secreted peptide containing two PDZ domains. EMBO Rep. 4, 412-418.

Zimmermann, P., 2006. The prevalence and significance of PDZ domainphosphoinositide interactions. Biochem. Biophys. Acta 1761, 947-956. 